\title{
ON FINITE INVARIANT MEASURES FOR MARKOV OPERATORS ${ }^{1}$
}

\author{
M. FALKOWITZ
}

\begin{abstract}
Two lemmas on proper vectors of convex linear combination of operators and semigroups in a Banach space are proved. They are applied to problems of invariant measures for Markov operators.
\end{abstract}

\section{Proper vectors of convex linear combinations.}

LEMMA 1. Let $\left\{P_{i}\right\}$ be commuting operators on the Banach space $B$ with $\left\|P_{i}\right\| \leqq 1$. Let $P=\sum_{i=1}^{\infty} \alpha_{i} P_{i}$ where $\alpha_{i}>0, \sum \alpha_{i}=1$. If $P x=\lambda x,|\lambda|=1$, then $P_{i} x=\lambda x, i=1,2, \cdots$.

Proof. Fix $i_{0}$; then

$$
P=\alpha_{i_{0}} P_{i_{0}}+\sum_{i \neq i_{0}} \alpha_{i} P_{i}=\alpha_{i_{0}} P_{i_{0}}+\left(1-\alpha_{i_{0}}\right) Q
$$

where $Q=\sum_{i \neq i_{0}}\left(\alpha_{i} /\left(1-\alpha_{i_{0}}\right)\right) P_{i}$; necessarily $\|Q\| \leqq 1$. By a lemma of Foguel [1, Lemma 2.1], $\left\|\left(P_{i_{0}}-Q\right) P^{n}\right\| \rightarrow_{n \rightarrow \infty} 0$. But

$$
\left\|\left(P_{i_{0}}-Q\right) P^{n} x\right\|=\left\|\lambda^{n}\left(P_{i_{0}} x-Q x\right)\right\|=\left\|P_{i_{0}} x-Q x\right\|,
$$

hence $P_{i_{0}} x=Q x$ and necessarily $P_{i_{0}} x=\lambda x$.

REMARK. The condition $\alpha_{i}>0$ is not essential in the lemma. If the $\alpha_{i}$ are nonzero and $\sum_{i=1}^{\infty}\left|\alpha_{i}\right|=|\lambda|$, the conclusion remains true, with $P_{i} x=$ $\left(\operatorname{sgn} \lambda / \operatorname{sgn} \alpha_{i}\right) x$. To see that, consider

$$
P=\sum_{i=1}^{\infty} \frac{\left|\alpha_{i}\right|}{|\lambda|}\left(\frac{\alpha_{i}}{\left|\alpha_{i}\right|} P_{i}\right) .
$$

Let us consider a strongly continuous semigroup of operators on $B$, $P_{t}$, with $\left\|P_{t}\right\| \leqq 1$. Given a measurable, nonnegative function $\phi(t), t \geqq 0$, with $\int^{\infty} \phi(t) d t=1$, define $R_{\phi}=\int_{0}^{\infty} \phi(t) P_{t} d t$; extend $\phi(t)$ to be zero for

Received by the editors July 3, 1972.

AMS (MOS) subject classifications (1970). Primary 28A65, 47A35, 60J 25.

Key words and phrases. Markov operators, invariant measures, proper vectors.

${ }^{1}$ This paper is part of the author's Ph.D. thesis prepared at the Hebrew University of Jerusalem under the supervision of Professor Foguel, to whom the author is grateful for his helpful advice and kind encouragement.

C) American Mathematical Society 1973 
$t<0$. For every $x \in B$, the function $\phi(t) P_{t} x$ is strongly measurable; see the proof of Theorem 9.2.2 in [5]. Since $\int_{0}^{\infty}\left\|\phi(t) P_{t} x\right\| d t<\infty$, it is Bochner integrable, and $\left\|R_{\phi}\right\| \leqq \int_{0}^{\infty} \phi(t) d t$ [5, Theorem 3.5.2].

We wish to find $R_{\phi} R_{\psi}$ :

$$
\begin{aligned}
\left\langle R_{\phi} R_{\psi} x, x^{*}\right\rangle & =\int_{0}^{\infty} \phi(t)\left\langle P_{t} R_{\psi} x, x^{*}\right\rangle d t \\
& =\int_{0}^{\infty} \phi(t)\left\langle R_{\psi} x, P_{t}^{*} x^{*}\right\rangle d t \\
& =\int_{0}^{\infty} \phi(t)\left(\int_{0}^{\infty} \psi(s)\left\langle P_{s} x, P_{t}^{*} x^{*}\right\rangle d s\right) d t \\
& =\int_{0}^{\infty} \phi(t)\left(\int_{0}^{\infty} \psi(s)\left\langle P_{s+t} x, x^{*}\right\rangle d s\right) d t .
\end{aligned}
$$

Changing variables, one obtains

$$
\begin{aligned}
\left\langle R_{\phi} R_{\psi} x, x^{*}\right\rangle & =\int_{0}^{\infty} \phi(t)\left(\int_{t}^{\infty} \psi(r-t)\left\langle P_{r} x, x^{*}\right\rangle d r\right) d t \\
& =\int_{0}^{\infty} \phi(t)\left(\int_{0}^{\infty} \psi(r-t)\left\langle P_{r} x, x^{*}\right\rangle d r\right) d t \\
& =\int_{0}^{\infty}\left(\int_{0}^{\infty} \phi(t) \psi(r-t) d t\right)\left\langle P_{r} x, x^{*}\right\rangle d r \\
& =\int_{0}^{\infty}(\phi * \psi)(r)\left\langle P_{r} x, x^{*}\right\rangle d r
\end{aligned}
$$

since Fubini's theorem certainly applies. Thus $R_{\phi} R_{\psi}=R_{\phi^{*} \psi}$.

Lemma 2. Let $R_{\phi}=\int_{0}^{\infty} \phi(t) P_{t} d t$ be the operator defined above. If $R_{\phi} x=\lambda x,|\lambda|=1$, then $\lambda=1$ and $P_{t} x=x, t \geqq 0$.

Proof. Suppose first that $\phi$ majorizes a positive multiple of the characteristic function of a certain interval. That is, there exist $c>0$ and $0 \leqq a<b$ such that $\phi \geqq c 1_{[a, b]}$. We choose $c$ small enough so that $c(b-a)<1$. Denote $1_{[a, b]}$ by $\chi$; then

$$
\begin{aligned}
R_{\phi} & =c R_{\chi}+R_{\phi-c \chi} \\
& =c(b-a)\left(R_{\chi} /(b-a)\right)+(1-c(b-a))\left(R_{\phi-c \chi} /(1-c(b-a))\right) .
\end{aligned}
$$

Since $\left\|R_{\chi} /(b-a)\right\|,\left\|R_{\phi-c x} /(1-c(b-a))\right\| \leqq 1$, the former lemma can be applied to get

$$
\frac{1}{b-a} \int_{a}^{b} P_{t} x d t=\lambda x .
$$


Now let $s_{0}, a<s_{0}<b$ and $\varepsilon>0$ be given. Let $\delta>0$ be such that $\left|s-s_{0}\right|<\delta \Rightarrow$ $\left\|P_{s} x-P_{s_{0}} x\right\|<\varepsilon$. If $s$ is also in the interval $\left(s_{0}, b\right)$, a repetition of the argument above shows

But

$$
\frac{1}{s-s_{0}} \int_{s_{0}}^{s} P_{t} x d t=\lambda x
$$

$$
\left\|\frac{1}{s-s_{0}} \int_{s_{0}}^{s} P_{t} x d t-P_{s_{0}} x\right\|<\varepsilon \text { for }\left|s-s_{0}\right|<\delta .
$$

Since $\varepsilon$ is arbitrary, $P_{s_{0}} x=\lambda x$. Thus $P_{t} x=\lambda x$ for all $a<t<b$. Now, for any $t>0$, let $n$ be so large that $t / n<b-a$; then, for a certain positive integer $k$, $a<k t / n<(k+1) t / n<b$. Hence

$$
\lambda x=P_{t / n}^{k+1} x=P_{t / n} P_{t / n}^{k} x=\lambda P_{t / n} x \quad \text { and therefore } P_{t} x=x .
$$

Necessarily $\lambda=1: \lambda x=P_{2 t} x=P_{t}^{2} x=\lambda^{2} x$.

For the case of a general $\phi$, we choose $0 \leqq \psi \leqq \phi$ bounded, so that $\psi * \psi$ is continuous (convolution of a $L_{1}$ function with a $L_{\infty}$ function; see [7, Theorem, p. 4]). Let

$$
\psi_{1}(t)=\left(\int_{0}^{\infty} \psi(s) d s\right)^{-1} \psi(t) .
$$

Then $R_{\psi_{1}} x=\lambda x$, implying $R_{\psi_{1^{*} \psi_{1}}} x=\lambda^{2} x$ and by the previous part, $P_{t} x=\lambda$ for all $t \geqq 0$ and $\lambda^{2}=1$. But then $\lambda x=R_{\phi} x=x$ and necessarily $\lambda=1$.

2. Application to Markov operators. Let $(x, \Sigma, m)$ be a finite measure space. We shall use the notation and definitions of [2].

Applied to Markov operators and invariant measures, Lemma 1 reads:

THEOREM 1. Let $P=\sum_{i=1}^{\infty} \alpha_{i} P_{i}$ where $P_{i}$ are commuting Markov operators, $\alpha_{i}>0$ and $\sum \alpha_{i}=1$. Then a finite invariant measure for $P$ is invariant for all $P_{i}$.

Remark. Suppose $P, Q$ are Markov operators, $P 1 \leqq Q 1$ and $P$ dominates $Q$ in the following sense: $P \geqq \alpha Q$ for some $0<\alpha<1$. Then $P=\alpha Q+(1-\alpha)(P-\alpha Q) /(1-\alpha)$ is a convex linear combination of Markov operators: clearly $(P-\alpha Q) /(1-\alpha)$ is positive and $((P-\alpha Q) /(1-\alpha)) 1 \leqq Q 1$ implies it is a contraction.

The following two results are known. Corollary 1 is due to S. Horowitz [4] (his result is slightly more general), and Corollary 2 to A. Brunel (unpublished). Let us show how to derive them from Theorem 1.

COROLlaRY 1. Let $\Pi$ be a commutative semigroup of Markov operators having no finite invariant measure equivalent to $m$. Then there exist $P_{i} \in \Pi$ and $\alpha_{i}>0, \sum \alpha_{i}=1$, such that $\sum_{i=1}^{\infty} \alpha_{i} P_{i}$ is not conservative. 
Proof. M. Lin has shown in [3] that $\inf _{P \in \Pi} m P(A)>0$ for every $A \in \Sigma$, $m(A)>0$, is a necessary and sufficient condition for a finite equivalent invariant measure for $\Pi$. Thus there exist a sequence $P_{i}$ such that there is no finite equivalent invariant measure common to all $P_{i}$. By Theorem 1 neither does any $Q=\sum_{i=1}^{\infty} \alpha_{i} P_{i}$ with $\alpha_{i}>0, \sum \alpha_{i}=1$, have such a measure. Brunel's result in [6] then supplies an operator $\sum_{j=0}^{\infty} \beta_{j} Q^{j}, \beta_{j} \geqq 0, \sum \beta_{j}=1$, which is not conservative. From the condition for conservativity in [8], $P h \leqq h$ for $0 \leqq h \leqq 1 \Rightarrow P h=h$, neither is $\left(1 /\left(1-\beta_{0}\right)\right) \sum_{j=1}^{\infty} \beta_{j} Q^{j}$, which is clearly a convex linear combination of members of II.

Corollary 2. Let $P$ be a Markov operator and $Q=\sum_{i=0}^{\infty} \alpha_{i} P^{i}$ where $\alpha_{i} \geqq 0, \sum \alpha_{i}=1$. Then an invariant measure $u$ for $Q$ is invariant for $P^{r}$, where $r$ is the greatest common divisor of $n>0$ such that $\alpha_{n}>0$.

Proof. There exist $n_{1}, \cdots, n_{k}$ with $\alpha_{n_{j}}>0$ and nonzero integers $q_{1}, \cdots, q_{k}$ such that $r=\sum_{j=1}^{k} q_{j} n_{j}$. Write $\sum_{1} q_{j} n_{j}$ for the summation over $q_{j}$ positive and $\sum_{2} q_{j} n_{j}$ for the summation over $q_{j}$ negative. Since, by Theorem $1, u P^{n_{j}}=u, j=1, \cdots, k$, we have

$$
u P^{r}=\left(u P^{-\Sigma_{2} a_{j} n_{j}}\right) P^{r}=u P^{\Sigma_{1} a_{j} n_{j}}=u .
$$

Let $\left\{P_{t}\right\}$ be a strongly continuous semigroup of Markov operators. Then Lemma 2 reads as follows:

THEOREM 2. A finite measure is invariant for $\left\{P_{t}\right\}$ if and only if it is invariant for any operator

$$
\int_{0}^{\infty} \phi(t) P_{t} d t, \quad \text { where } \phi(t) \geqq 0, \quad \int_{0}^{\infty} \phi(t) d t=1 .
$$

Using Brunel's result in [6] we may conclude:

COROLlaRY. If $\left\{P_{t}\right\}$ has no m-equivalent finite invariant measure, then there exists a function $\phi(t)$ with $\phi(t) \geqq 0, \int_{0}^{\infty} \phi(t) d t=1$, such that $\int_{0}^{\infty} \phi(t) P_{t} d t$ is not conservative.

Indeed, for any $R_{\psi}=\int_{0}^{\infty} \psi(t) P_{t} d t$, if it is conservative, there are $\alpha_{n} \geqq 0$, $\sum \alpha_{n}=1$, such that $\sum \alpha_{n} R_{\psi}^{n}$ is not conservative. Put $\phi(t)=\sum \alpha_{n}\left(\phi^{*}\right)^{n}$.

ADDED IN PROOF. Lemma 2 (and Theorem 2) hold for the general case of a strongly continuous operator representation by operators of norm 1 , of a locally compact connected and metrizable Abelian group. Proofs are virtually the same, with necessary modifications.

\section{REFERENCES}

1. S. R. Foguel, On convex power series of a conservative Markov operator (to appear).

2. - The ergodic theory of Markov processes, Van Nostrand Math. Studies, no. 21, Van Nostrand, Princeton, N.J., 1969. MR 41 \#6299. 
3. M. Lin, Semi-groups of Markov operators (to appear).

4. S. Horowitz, On finite invariant measures for sets of Markov operators, Proc. Amer. Math. Soc. (to appear).

5. E. Hille, Functional analysis and semi-groups, Amer. Math. Soc. Colloq. Publ., vol. 31, Amer. Math. Soc., Providence, R.I., 1948. MR 9, 594.

6. A. Brunel, New conditions for existence of invariant measures in ergodic theory, Contributions to Ergodic Theory and Probability (Proc. Conf. Ohio State Univ., Columbus, Ohio, 1970), Springer-Verlag, Berlin and New York, 1970, pp. 7-17. MR 42 \#3253.

7. Walter Rudin, Fourier analysis on groups, Interscience Tracts in Pure and Appl. Math., no. 12, Interscience, New York, 1962. MR 27 \#2808.

8. S. R. Foguel, Remarks on conservative Markov processes, Israel J. Math. 6 (1968), 381-383. MR 39 \#4939.

Department of Mathematical Sciences, Tel Aviv University, Tel Aviv, Israel 